\title{
Solving fractional circuits with sinusoidal sources by using the gcdAlpha method
}

\author{
Marcin Sowa ${ }^{1, *}$ \\ ${ }^{1}$ Silesian University of Technology, Faculty of Electrical Engineering, Akademicka 2A, 44-100 Gliwice, Poland
}

\begin{abstract}
This paper concerns a study being part of a larger project aiming at solutions of problems with fractional time derivatives. The presented study concerns gcdAlpha - a semi-analytical method for solving fractional differential equations. The basis of the method is recalled along with the general form of problems it was designed to solve. Sources represented by sinusoidal time functions are considered and the general formulae for gcdAlpha are presented for this case. An exemplary circuit problem (containing fractional elements and a sinusoidal source) has been brought forward and solved. The results are compared with ones obtained through a solver basing on the numerical method called SubIval.
\end{abstract}

\section{Introduction}

Fractional calculus [1] is a quite popular field of late, where the concepts of fractional derivatives (and fractional integrals) are tested through their applications in various fields, e.g. circuit analyses [2] and control theory [3].

The introduction of fractional coils and fractional capacitors as elements in a circuit problem is associated with complications when it comes to solutions. The possibilities for obtaining solutions are: evaluations of analytical solutions [4], the use of semi-analytical methods [5] and numerical methods [6]. Numerical methods allow to solve the widest variety of problems. The author's main research revolves around SubIval - a numerical method. The acronym comes from how it has been named when it first appeared in [7], i.e. "the subinterval-based method".

The paper concerns a secondary project aiming at the design of methods, by which referential solutions can be obtained, which can be later applied when designing SubIval and its improvements.

The analysis is limited to problems with the Caputo definition of the fractional derivative [8] (of order $\alpha \in(0,1])$ :

$$
{ }_{0} \mathrm{D}_{t}^{\alpha} x(t)=\frac{1}{\Gamma(1-\alpha)} \int_{0}^{t} \frac{x^{(1)}(\tau)}{(t-\tau)^{\alpha}} \mathrm{d} \tau
$$

\section{The method details}

The gcdAlpha method, appearing in the title of this paper, is a semi-analytical method able to solve fractional differential equations (FDE) of the form:

$$
\mathbf{d}^{\alpha} \boldsymbol{x}(t)=\boldsymbol{A} \boldsymbol{x}(t)+\boldsymbol{B} \boldsymbol{v}(t),
$$

with $\boldsymbol{x}(t)$ being the vector of $N$ state variables, $\boldsymbol{v}(t)$ being the vector of $M$ source time functions and $\mathbf{d}^{\alpha} \boldsymbol{x}(t)$ being the vector of the fractional derivatives (i.e. the $i$-th entry is the fractional derivative of $x_{i}(t)$ of order $\left.\alpha_{i}\right)$.

For a more detailed description of the basics of the method the reader is referred to the previous paper, where the method was first introduced [9].

The name of the method was inspired by its first characteristic feature, where the greatest common divisor is found:

$$
\operatorname{gcd}\left(\alpha_{1}, \alpha_{2}, \alpha_{3}, \ldots \alpha_{N}\right)=\alpha .
$$

This allows the problem in the form of (2) to be expressed in a form:

$$
\mathbf{d}^{\alpha} \boldsymbol{w}(t)=\boldsymbol{C} \boldsymbol{w}(t)+\boldsymbol{D} \boldsymbol{v}(t),
$$

with only one derivative order $\alpha$. There is a welldescribed procedure [9] on how to obtain $\boldsymbol{C}$ and $\boldsymbol{D}$ from $\boldsymbol{A}$ and $\boldsymbol{B}$. The first entries of $\boldsymbol{w}(t)$ contain the actual solution vector $\boldsymbol{x}(t)$.

The solution for $\boldsymbol{w}(t)$ can be given as:

$$
\boldsymbol{w}(t)=\sum_{k=0}^{\infty} \frac{\boldsymbol{C}^{k} t^{k \alpha}}{\Gamma(k \alpha+1)} \boldsymbol{w}\left(t_{0}\right)+\sum_{k=0}^{\infty} \frac{\boldsymbol{C}^{k}}{\Gamma((k+1) \alpha)}\left[\begin{array}{c}
\mathbf{0}_{\sigma-N} \\
\boldsymbol{S}_{(k)}(t)
\end{array}\right],(5)
$$

where the notation $\mathbf{0}_{n}$ is applied for vectors of $n$ zeros ; $\boldsymbol{S}_{(k)}(t)$ is a special notation for the integral:

$$
\boldsymbol{S}_{(k)}(t)=\int_{0}^{t}(t-\tau)^{(k+1) \alpha-1} \boldsymbol{B} \boldsymbol{v}(t) \mathrm{d} \tau .
$$

The entries for the vector are given by:

$$
S_{(k), i}(t)=\sum_{j=1}^{M} B_{i, j} a_{(k), j}(t) . \quad i=1,2, \ldots N,
$$

where:

$$
a_{(k), j}(t)=\int_{0}^{t}(t-\tau)^{(k+1) \alpha-1} v_{j}(\tau) \mathrm{d} \tau .
$$

has been introduced in order to separately take into account the integrals appearing in the solution.

This is the second characteristic feature of gcdAlpha - for general forms of the source time functions $v_{j}(t)$ one can determine the forms of $a_{(k), j}(t)$ so that the integration 
can be handled analytically. In [9] the forms of $a_{(k), j}(t)$ have been determined and tested for step functions; this paper concerns their form for $v_{j}(t)$ being sinusoidal functions of the form:

One can compute:

$$
v_{j}(t)=v_{j \mathrm{~A}} \sin \left(\omega_{j} t+\varphi_{j}\right) .
$$

$$
\underline{a}_{(k), j}(t)=\left(\mathrm{j} \omega_{j}\right)^{-\alpha(k+1)} v_{j \mathrm{~A}} \mathrm{e}^{\mathrm{j}\left(\omega_{j} t+\varphi_{j}\right)} \gamma\left(\alpha(k+1), \mathrm{j} \omega_{j} t\right),
$$

whose imaginary part is $a_{(k), j}(t)$; in (10) one can notice the lower incomplete gamma function:

$$
\gamma(s, z)=\int_{0}^{z} t^{s-1} \exp (-t) \mathrm{d} t .
$$

For the computation of this function one can apply the formula in [10] applying Gauss's continued fraction.

\section{Computational example}

The simple example depicted in Fig. 1 has been considered.

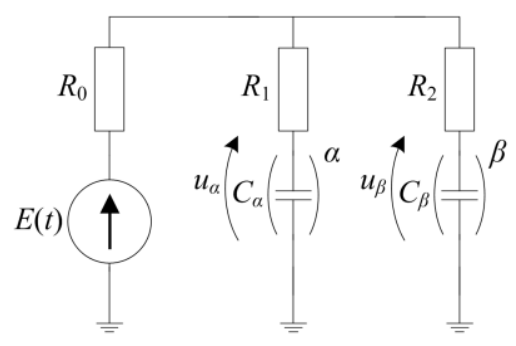

$$
\begin{aligned}
& E(t)=E_{\max } \sin (\omega t) \\
& R_{0}=0.9 \Omega \\
& R_{1}=0.5 \Omega \\
& R_{2}=0.7 \Omega \\
& E_{\max }=20 \mathrm{~V} \\
& \omega=2 \pi f \\
& f=50 \mathrm{~Hz} \\
& \alpha=0.9 \\
& C_{\alpha}=1 \mathrm{~F} \cdot \mathrm{s}^{1-\alpha} \\
& \beta=0.6 \\
& C_{\beta}=2 \mathrm{~F} \cdot \mathrm{s}^{1-\beta}
\end{aligned}
$$

Fig. 1. Exemplary circuit example with a sinusoidal source and fractional elements.

The state variables for the problem are the fractional capacitor voltages. The obtained time functions for $t \in[0,4 / f]$ are depicted in Fig. 2 .
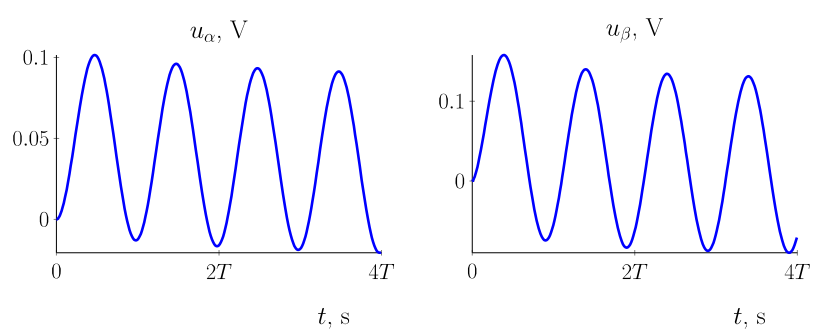

Fig. 2. Fractional capacitor voltage time functions obtained through the gedAlpha method.

The result has been compared with one obtained through a numerical solver basing on SubIval [11]. There are no visible differences between the results when plotting them in the same figure. The error is computed through the formula:

$$
e_{j}=100 \cdot \frac{\left|x\left(t_{j}\right)-x_{j}\right|}{\max _{j=1,2, \ldots n_{t}}\left|x\left(t_{j}\right)\right|} \%,
$$

with $x$ being the considered variable, $t_{1}, t_{2}, \ldots t_{n_{t}}$ being the time instances selected by the numerical solver, $x\left(t_{j}\right)$ are the values obtained through gedAlpha and $x_{j}$ is the numerical solution for $x$ at $t_{j}$. The averages of these error values are $1.62 \cdot 10^{-3} \%$ for $u_{\alpha}$ and $4.31 \cdot 10^{-4} \%$ for $u_{\beta}$.

The most important parameters selected for the SubIval solver are: $p=4$ (the maximum applied approximating polynomial order) and $e_{\text {ctrl }}=0.01 \%$ (the desired error for the computations of the fractional derivative).

\section{Conclusions}

The semi-analytical method gcdAlpha has been recalled. The basis of the method has been briefly described. In the previous work the method has been tested for unit step sources. With the study presented in this research the method now allows to solve problems where sinusoidal sources appear.

Other forms of the source time functions can also be supported - it all comes down to computing a general, analytical formula for the integral (8), e.g. as was given in (10) for sources in the form of (9).

\section{References}

1. KB. Oldham, J. Spanier, The Fractional Calculus, Academic Press, New York (1974)

2. W. Mitkowski, P. Skruch, Bull. Pol. Ac.: Tech. 61, 3 (2013)

3. A. Dabiri, B.P. Moghaddam, J.A. Tenreiro Machado, J. Comput. Appl. Math. 339 (2018)

4. T. Kaczorek, Fractional linear systems and electrical circuits, Springer (2015)

5. N.S. Khodabakhshi, S.M. Vaezpour, D. Baleanu, Fract. Calc. Appl. Anal. 17, 2 (2014)

6. R. Garrappa, J. Comput. Appl. Math. 276 (2016)

7. M. Sowa, Bull. Pol. Acad. Sci.: Tech. 62, 3 (2014)

8. M. Caputo, Geophys. J. Int. 13, 5 (1967)

9. M. Sowa, Computer Applications in Electrical Engineering 96 (2018)

10. M. Duckett, Fourier Series and Gamma Function, (Learning Press, New York, 2016)

11. M. Sowa, Springer, (2019) 